\title{
Local ionospheric electron density profile reconstruction in real time from simultaneous ground-based GNSS and ionosonde measurements
}

\author{
S. M. Stankov ${ }^{1, *}$, K. Stegen ${ }^{1}$, P. Muhtarov ${ }^{2}$, R. Warnant ${ }^{1}$ \\ ${ }^{1}$ Royal Meteorological Institute (RMI), Ringlaan 3, B-1180 Brussels, Belgium \\ ${ }^{2}$ Geophysical Institute, Bul. Acad. Sciences, G. Bonchev Str. 3, 1113 Sofia, Bulgaria \\ * Corresponding author. Tel.: +32 60395 472; Fax: +32 60395 423; E-mail: S.Stankov@ meteo.be
}

\begin{abstract}
The purpose of the LIEDR (local ionospheric electron density profile reconstruction) system is to acquire and process data from simultaneous ground-based total electron content (TEC) and digital ionosonde measurements, and subsequently to deduce the vertical electron density distribution above the ionosonde's location. LIEDR is primarily designed to operate in real time for service applications and, for research applications and further development of the system, in a post-processing mode. The system is suitable for use at sites where collocated TEC and digital ionosonde measurements are available. Developments, implementations, and some preliminary results are presented and discussed in view of possible applications.
\end{abstract}

Keywords: ionosphere, TEC, electron density, critical frequency, plasma frequency.

\section{Introduction}

Since the early 1990s, the needs for real-time ionospheric measurements and updates to climatological ionospheric models have been constantly increasing, driven primarily by requirements for corrections to precise satellite ranging systems. These needs, of users who may have their systems affected by the ionosphere, include (Klobuchar, 1997): ionospheric range delay estimations in satellite surveillance and positioning systems, scintillation predictions for satellite communications, radio frequency communications in general, and various research applications.

Real-time ionospheric data provision from modern digital ionosondes offers important input to address the abovementioned needs. Nowadays, the digisonde is a powerful tool in ionospheric nowcast because of its improved reliability, accuracy and precision, its ability to automatically scale and analyse the sounding data, and also to promptly distribute the results through internet connections. However, studies have pointed at some deficiencies. For example, a premature truncation of the scaled F2 trace were made by earlier versions of the autoscaling software, caused by echo trace gaps due to restricted frequency bands and/or bands of strong interference (Reinisch et al., 2005). Also, a comparison of the true-height electron density profiles inverted from ionograms using the POLAN (Titheridge, 1988) and NHPC (Reinisch et al., 2005) methods showed significant systematic differences (Sauli et al., 2007). Such differences can be explained with the differences in the underlying ionospheric profilers that are used. In fact, a major issue is the determination of the topside electron density profile (Fig.1A) because the ground-based ionosonde only provides observations on the bottom-side ionosphere. In the case of the Lowell digisonde (Reinisch et al., 2005), the topside profile is automatically calculated based on a technique assuming $\alpha$-Chapman distribution (Fig.1B) with a scale height that is derived from the measured bottomside profile. An advantage is that the Chapman profiler needs only the F2 peak electron density characteristics and the scale height values to calculate the topside distribution. A disadvantage is the assumption of (constant) topside scale height equal to that immediately below the peak. Besides, there are other ionospheric profilers that might be more suitable in different spatial and temporal conditions (Stankov, 2002b) and that normally produce quite different altitudinal distributions in the F-region (Fig.1B). There are, however, several ongoing efforts for improving the presentation of ionospheric sounding data by developing more robust techniques for automated scaling, providing statistical/quality estimates for each autoscaled parameter/result, and deriving value-added products from the sounding data that require less or no manual interpretation (Reinisch et al., 2005). These efforts include also modeling the topside electron density by an $\alpha$-Chapman function, with continuously varying scale height, fitted to IMAGE/RPI and ISIS topside measurements (Reinisch et al., 2007). 

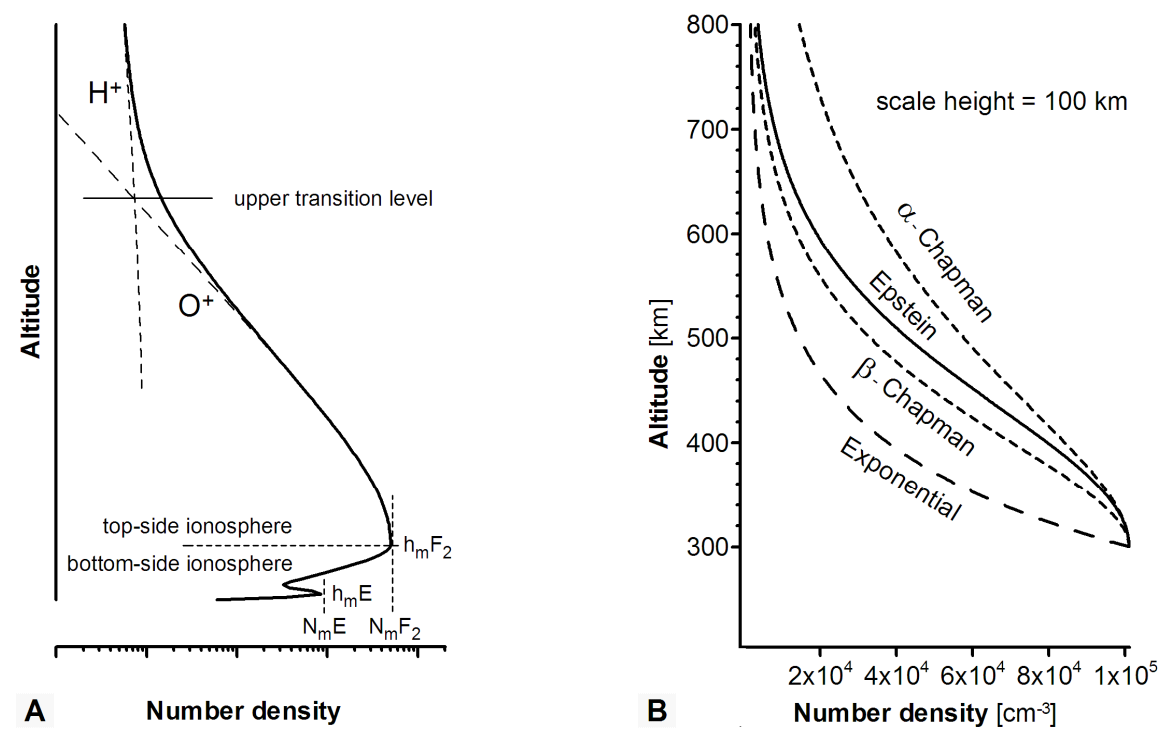

Fig.1. Ion and electron density profile characteristics (panel A). Comparison between vertical electron density profiles obtained with basic analytical models for a given scale height of $100 \mathrm{~km}$ (panel B).

Various data ingestion and assimilation approaches/techniques are being tried in order to develop the next generation of ionospheric modeling and nowcast (e.g. Schunk et al., 2004; Stamper et al., 2004; Buresova et al., 2009; and the references therein). Albeit relying heavily on ionosonde measurements, all of them incorporate additional data, being from Global Navigation Satellite System (GNSS), topside sounders, satellite in-situ, or ionospheric radio occultation measurements.

The paper presents an operational system for deducing the vertical distribution of the electron density in the local ionosphere. The system, dubbed LIEDR (Local Ionospheric Electron Density profile Reconstruction), acquires and processes in real time the concurrent and collocated groundbased TEC and digital ionosonde measurements, and ultimately, deduces a full-height electron density profile based on a reconstruction technique proposed by Stankov et al. (2003). In this way, the topside profile is more adequately represented because of the use of additional information about the topside ionosphere, such as TEC and $\mathrm{O}^{+} / \mathrm{H}^{+}$ion transition height.

The paper is organised as follows. First, the reconstruction method will be outlined together with the database and measurements that are required. Next, the LIEDR system layout and implementation will be described. Exemplary results will be presented and discussed in the next part. The paper will conclude with an outlook for further developments and possible applications.

\section{Method}

The vertical electron density profile at a given location is deduced from ground-based measurements of the total electron content, ionospheric vertical soundings, and empirically-obtained values of the upper ion transition height/level (UTL) - the height at which the $\mathrm{O}^{+}$and $\mathrm{H}^{+}$ion densities are equal. The retrieval of the corresponding electron density distribution is performed in two main stages: construction of the bottom-side electron profile (i.e. below the F2-layer density peak height, $h_{m} F_{2}$ ) and construction of the top-side profiles (above $h_{m} F_{2}$ ). Here we will only outline the reconstruction technique. More details, including the derivation of all formulae, can be found in Stankov et al. (2003).

High-precision ionosonde measurements are used for directly obtaining the lower part of the electron density profile based on Epstein layer functions utilizing the measured values of the critical frequencies, $f_{o} F_{2}$ and $f_{o} E$, the peak height, $h_{m} F_{2}$, and the propagation factor, $M_{3000} F_{2}$ (Rawer, 1983, 1988; Di Giovanni and Radicella, 1990). The corresponding bottom-side part of TEC is 
calculated from this profile and is then subtracted from the entire TEC in order to obtain the unknown portion of TEC in the upper side (i.e. above $\left.h_{m} F_{2}\right)$. The topside TEC $\left(T E C_{\text {top }}\right)$ is used in the next stage for deducing the top-side ion and electron profiles.

The electron density $\left(N_{e}\right)$, at a given altitude $h$ in the topside ionosphere, is considered as a sum of the constituent major ions $\left(\mathrm{O}^{+}\right.$and $\left.\mathrm{H}^{+}\right)$densities, $N_{O+}$ and $N_{H+}$, i.e.

$$
N_{e}(h)=N_{O+}(h)+N_{H+}(h)
$$

Each ion density profile is permitted to take one of several forms, as follows:

(Exponential): $\quad N_{i}(h)=N_{i}\left(h_{m}\right) \exp \left\{-\frac{h-h_{m}}{H_{i}}\right\}$

(Chapman): $\quad N_{i}(h)=N_{i}\left(h_{m}\right) \exp \left\{c\left[1-\frac{h-h_{m}}{H_{i}}-\exp \left(-\frac{h-h_{m}}{H_{i}}\right)\right]\right\}, c=\left\{\begin{array}{l}0.5, \alpha-\text { Chapman } \\ 1.0, \beta-\text { Chapman }\end{array}\right.$

(Epstein, sech-squared): $\quad N_{i}(h)=N_{i}\left(h_{m}\right) \operatorname{sech}^{2}\left(\frac{h-h_{m}}{2 H_{i}}\right)$

where $N_{i}$ and $H_{i}$ are the corresponding ion's $\left(\mathrm{O}^{+}\right.$or $\left.\mathrm{H}^{+}\right)$density and scale height, and $h_{m}$ is the maximum ion density height which, for both ions, is assumed to be at the height of the electron density peak, $h_{m} F_{2}$. Thus, considering the two major ions $\left(\mathrm{O}^{+}\right.$and $\left.\mathrm{H}^{+}\right)$in the topside ionosphere, there are four parameters that need to be determined in order to reconstruct the topside electron density profile. These parameters are the oxygen topside scale height $\left(H_{O_{+}}\right)$, the oxygen concentration at $h_{m}\left(N_{m O_{+}}\right)$, the hydrogen topside scale height $\left(H_{H_{+}}\right)$, and the hydrogen concentration at $h_{m}\left(N_{m H_{+}}\right)$. These 4 unknowns require a system of 4 equations in order to find a unique solution. For the purpose, the following system is assembled:

$$
\begin{aligned}
& N_{m O+}+N_{m H+}=N_{m} \\
& H_{H+}=\left(\mu_{O+} / \mu_{H+}\right) \xi H_{O+} \\
& T E C_{t o p}=\aleph_{O+}\left(H_{O+}, N_{m O+}, h_{m}\right)+\aleph_{H+}\left(H_{H+}, N_{m H+}, h_{m}\right) \\
& N_{O+}\left(H_{O+}, N_{m O+}, h_{m} ; h_{t r}\right)=N_{H+}\left(H_{H+}, N_{m H+}, h_{m} ; h_{t r}\right)
\end{aligned}
$$

where:

$$
\begin{aligned}
& N_{m}-\text { the } \mathrm{F} 2 \text {-layer peak electron density }\left(N_{m} F_{2}\right) \\
& \mu_{O+}-\text { the } \mathrm{O}^{+} \text {ion mass } \\
& \mu_{H_{+}}-\text {the } \mathrm{H}^{+} \text {ion mass } \\
& \xi-\text { the vertical 'scale height' corrector, } \xi=\sin [\arctan (2 \tan \varphi)], \varphi \text { - latitude } \\
& h_{t r}-\text { the } \mathrm{O}^{+} / \mathrm{H}^{+} \text {ion transition height } \\
& \left.T E C_{t o p}-\text { the measured topside TEC (content above } h_{m} F_{2}\right) \\
& \aleph_{O+}-\text { the integrated topside } \mathrm{O}^{+} \text {ion concentration } \\
& \aleph_{H_{+}}-\text {the integrated topside } \mathrm{H}^{+} \text {ion concentration }
\end{aligned}
$$

The first system equation (5), represents the principle of plasma quasi-neutrality. The next equation (6) represents the relation between the $\mathrm{O}^{+}$and $\mathrm{H}^{+}$ion scale heights. The third equation (7) is obtained after integrating the proposed 'reconstruction' formula (1) from $h_{m} F_{2}$ to infinity. The last equation 
(8) summarizes the fact that the $\mathrm{O}^{+}$and $\mathrm{H}^{+}$ion densities are equal at the upper ion transition level. The system is solved by excluding the unknowns from the first three equations and replacing them in the last equation. The resulting transcendental equation features only one unknown parameter, $\mathrm{H}_{\mathrm{O}_{+}}$, provided that the values of $T E C_{t o p}$ (also denoted with $\Phi_{t}$ ), $h_{m}$, and $h_{t r}$ are already known measured/modelled (see Section 3 Data and measurements). The transcendental equations, corresponding to each of the above-described profilers $(2,3,4)$ are, correspondingly:

$$
\begin{aligned}
& \left(\frac{16 \xi}{(16 \xi-1)} N_{m}-\frac{1}{(16 \xi-1) H_{O+}} \Phi_{t}\right) \exp \left(-\frac{h_{t r}-h_{m}}{H_{O+}}\right)- \\
& -\left(\frac{1}{(16 \xi-1) H_{O+}} \Phi_{t}-\frac{1}{(16 \xi-1)} N_{m}\right) \exp \left(-\frac{h_{t r}-h_{m}}{16 \xi H_{O+}}\right)=0 \\
& \left(\frac{16 \xi}{(16 \xi-1)} N_{m}-\frac{1}{\hat{c}(16 \xi-1) H_{O+}} \Phi_{t}\right) \times \exp \left(1-\frac{h_{t r}-h_{m}}{H_{O+}}-\exp \left(-\frac{h_{t r}-h_{m}}{H_{O+}}\right)\right)- \\
& -\left(\frac{1}{\hat{c}(16 \xi-1) H_{O+}} \Phi_{t}-\frac{1}{(16 \xi-1)} N_{m}\right) \times \exp \left(1-\frac{h_{t r}-h_{m}}{16 \xi H_{O+}}-\exp \left(-\frac{h_{t r}-h_{m}}{16 \xi H_{O+}}\right)\right)=0 \\
& \left(\frac{16 \xi}{(16 \xi-1)} N_{m}-\frac{1}{2(16 \xi-1) H_{O+}} \Phi_{t}\right) \operatorname{sech}^{2}\left(\frac{h_{t r}-h_{m}}{2 H_{O+}}\right)- \\
& -\left(\frac{1}{2(16 \xi-1) H_{O+}} \Phi_{t}-\frac{1}{(16 \xi-1)} N_{m}\right) \operatorname{sech}^{2}\left(\frac{h_{t r}-h_{m}}{32 \xi H_{O+}}\right)=0
\end{aligned}
$$

Each of these equations can be solved using dichotomy methods for finding the root (i.e. the unknown). Having the $\mathrm{O}^{+}$scale height value $H_{O+}$ calculated, the rest of the unknowns ( $H_{H+}, N_{m O+}, N_{m H+}$ ) can be obtained from formulae (5) - (7). The topside electron profile is then easy to reconstruct from the main formula (1) and the selected profiler.

A similar method for electron profile reconstruction has been developed based on ionospheric radio occultation measurements of the over-satellite electron content (Stankov et al., 2005).

\section{Data and measurements}

Most measurements needed for the LIEDR operation are made at the RMI Geophysical Centre in Dourbes $\left(50.1^{\circ} \mathrm{N}, 4.6^{\circ} \mathrm{E}\right)$ (Jodogne and Stankov, 2002).

The Dourbes digital ionosonde (URSI code: DB049) carries out regular vertical ionospheric soundings with a Digisonde 256 sounder, developed by the University of Massachusetts - Lowell (Reinisch, 1996). All ionograms are automatically scaled and the values of $f_{o} F_{2}, f_{o} E, M_{3000} F_{2}$, $h_{m} F_{2}$ are deduced with short delay of a couple of minutes. Some ionogram settings are as follows: frequency range 1-16 MHz, frequency scale - linear, frequency step - $100 \mathrm{kHz}$, amplitude resolution $0.25 \mathrm{~dB}$, phase resolution $-1.4^{\circ}$, Doppler resolution $-4 \mathrm{~Hz}$, range resolution $-5 \mathrm{~km}$, range start -60 $\mathrm{km}$, ionogram duration $-4 \mathrm{~min}$. The sounding rate is set to 1 per every 15 minutes, but it can be increased if required.

The TEC observations are made with a GPS (Global Positioning System) receiver (co-located with the digisonde) by applying a computational procedure based on a 'geometry-free' combination of GPS code and phase measurements for resolving the ambiguities (Warnant and Pottiaux, 2000). Receiver and satellite group delays are estimated via polynomial approximation of the slant TEC, 
depending on latitude and local time. The conversion to vertical TEC assumes the standard ionospheric thin-shell model at a mean ionospheric height of $350 \mathrm{~km}$. To obtain a TEC value representative of the ionosphere above a given location, selected and averaged are all values within a latitudinal range of $\pm 1.5^{\circ}$ over a 15 minute period.

It is well known that the plasma scale height, and the vertical plasma distribution in general, change substantially during active/storm geomagnetic conditions (e.g. Hargreaves, 1992; Rees and Fuller-Rowell, 1989; Stankov and Jakowski, 2006). From this aspect, monitoring the local geomagnetic activity is considered an important component of the LIEDR system. First, simultaneously monitoring the ionospheric and geomagnetic activity facilitates the analysis/interpretation of the LIEDR nowcast and the investigation of local ionospheric phenomena. Second, the geomagnetic activity index is a key driver of the ionospheric forecast being currently developed as an extension of LIEDR. For determination of the local geomagnetic activity, we use 1min vector magnetic field ( $\mathrm{H}, \mathrm{D}$, and $\mathrm{Z}$ components) data as obtained directly from the instruments of the Dourbes magnetic observatory; with precision of $1 \mathrm{sec}$ for time and $0.1 \mathrm{nT}$ for the field components. A nowcast system for operational estimation (every one hour) of the geomagnetic index $\mathrm{K}$ has already been deployed. The system is based on a fully automated computer procedure for realtime digital magnetogram data acquisition, dataset screening and removing of outliers, establishing the solar regular $\left(\mathrm{S}_{\mathrm{R}}\right)$ variation of the geomagnetic field, calculating the $\mathrm{K}$ index, and optionally, issuing an alert if storm-level activity is indicated.

Since the relative abundance of oxygen and hydrogen ions in the upper ionosphere is a significant factor affecting the topside plasma density distribution, the $\mathrm{O}^{+} / \mathrm{H}^{+}$transition height is considered a key reference point in the reconstruction technique. Such an "anchor" point is particularly useful because it can be determined from independent (e.g. space-based) measurements (improving the reliability of the profile reconstruction method) and it is always above the $\mathrm{F}$ layer peak height (ensuring the robustness of the computational procedure). Here, the UTL value is obtained from a previouslydeveloped empirical model (Kutiev et al., 1994; Stankov, 2002a), based on various satellite and rocket in-situ measurements (of the individual $\mathrm{O}^{+}$and $\mathrm{H}^{+}$ion densities), parameterised by solar activity, season, local time, latitude and longitude. Thus, effectively, the model output is an (multi-variable polynomial) approximated quantity, providing quick and convenient reference value with respect to any eligible set of the above-listed input parameters.

\section{Implementation}

The purpose of the LIEDR system is to acquire and process data from simultaneous ground-based GNSS TEC and digital ionosonde measurements, and subsequently to deduce the vertical electron density distribution in the local ionosphere. The system has a modular structure (Fig.2) allowing for easier maintenance. The main modules/facilities are: System Administration, Instruments and Measurements, Database Management, Software and Models, Data Processing, Communication and Dissemination.

The System Administration is responsible for the overall control of the LIEDR operation, including: software monitoring and upgrading, data acquisition, processing and storage, timing, product display and dissemination, communication with the user, etc.

The Timer controls the start of the data acquisition and processing as well as holding the current time information. Nominally, the time resolution between two consecutive reconstructions is set to 15 minutes. Data from the digital ionosonde is available shortly after a sounding is taken; a greater delay ( 10 min) is therefore typically caused by the need for sufficient measurements and processing to derive the GPS TEC value. All data transfer is based on the File Transfer Protocol (FTP).

Four types of measurements are needed for the operation of LIEDR: vertical incidence soundings, TEC, geomagnetic and solar activity. Vertical incidence soundings are performed with a digital ionosonde DGS-256 (Lowell). TEC measurements are obtained from a GPS receiver collocated with the digisonde. The local geomagnetic activity is determined via a newly developed nowcast system based on measurements from the Dourbes magnetic observatory. A reserve option is the Kp nowcast (Kutiev et al., 2009) produced from solar wind observations by the Advanced Composition Explorer (ACE) satellite. For the solar activity estimation, the F10.7 index nowcast is used (SIDC/NOAA). 


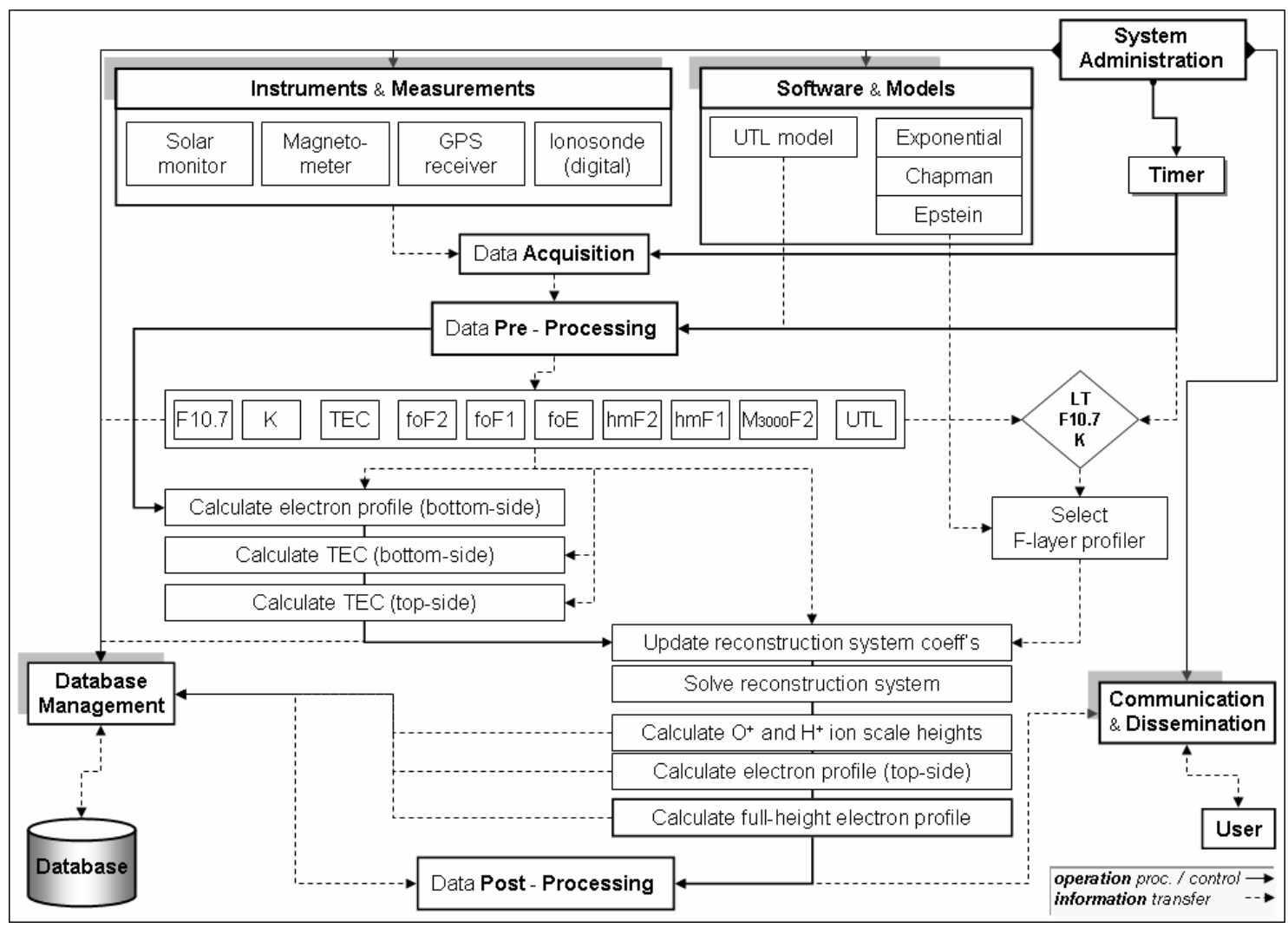

Fig.2. Layout of the operational LIEDR system. The operational control chain is represented with a solid line while the data/information transfer - with dashed lines. Direction/s of control/transfer shown with arrow/s.

The access to the LIEDR database is arranged only through the Database Management unit. Currently, the database consists of ionosonde, GPS TEC, solar and geomagnetic indices records for the period since year 1993. It is constantly being upgraded with the most recent measurement data and products of the profile reconstruction.

The system's programs are coded in C, FORTRAN, R and PHP languages. As mentioned earlier, the UTL is obtained from an empirical model (Kutiev et al., 1994; Stankov, 2002a) embedded in the reconstruction software.

The Data Processing module includes pre-processing, processing and post-processing units. The pre-processing starts immediately after the data acquisition (for the current run) is completed. Following a thorough data screening process (removing outliers and determining whether fallback substitutes are needed), all input parameters for the profile reconstruction procedure (such as TEC, $f_{o} F_{2}, h_{m} F_{2}$, UTL, etc.) are calculated and prepared for the next step. The main processing follows strictly the algorithm for profile reconstruction: calculating the bottom- and top-side TEC values, updating the coefficients and solving the reconstruction system of equations, computing the unknown topside ion scale heights, and finally deducing the full-length electron density profile. In doing so, there is an opportunity for selecting the most appropriate top-side profiler given the current local time, solar and geomagnetic activity. Evaluations of these different forms of top-side profiles revealed that the Exponential layer was the best representation of day-time conditions, with the Epstein or Chapman layers giving better results for night-time (Stankov, 2002b). As mentioned already, if some observations are not available in time, there are possibilities for fallback substitutes for some inputs, $f_{o} E$ and $M_{3000} F_{2}$ in particular (Stankov et al., 2005). For research applications and further development of the system, a post-processing mode of operation is also envisaged. It allows for reproducing the electron density distribution from final input data records, for example the critical frequencies and the $\mathrm{K}$ index from manually-corrected ionograms and magnetograms, respectively. 
The Communication and Dissemination (CDF) unit is responsible for the products display, alert activation, and interaction with the user. CDF offers opportunities for viewing current plots and results, obtaining historical data records and plots, setting alert preferences, and communicating with the system administrators concerning all aspects of the LIEDR services.

\section{Results and discussion}

The described nowcast system has been tested on actual measurements at the RMI Dourbes and it proved to be capable of producing density profiles every 15 min using new observations, which is a sufficiently good rate for most of the envisaged applications, including ionospheric storm investigations.

As an exemplary demonstration, a screenshot of the LIEDR system output is presented in Fig.3 for the recent magnetic storm period of 5-7 April 2010. During that period, the solar activity was low $(76 \leq F 10.7 \leq 79)$ and the real-time estimates of the geomagnetic indices (Fig.3, bottom panels) indicated a Class-II storm ( $\max K=6$, min Dst $=-87$ ). In the top panel of Fig.3, the reconstructed altitudinal electron density profile is plotted against the universal time as soon as the profile becomes available. Thus, over time, a so-called profilogram is produced, in this case over a period of 96 hours. For better graphic representation, instead of the electron density $\left(N_{e}\right)$, the plasma frequency $\left(f_{p}\right)$ is

plotted (colour-coded, scale on the right): $f_{p}[M H z]=0.898 \times 10^{-5} \sqrt{N_{e}\left[e l / m^{3}\right]}$. In this case, the Epstein layer is applied for night-time conditions, while the Exponential profiler is used during daytime hours. Note the detailed vertical distribution in the upper ionosphere - it is easy to detect the changes in the calculated scale height and the resulting density distribution as they change during the day.

The input values of the GPS-derived TEC, together with the vertical sounding measurements $f_{o} F_{2}$ and $f_{o} E$, are given in Fig.3B. TEC and $f_{o} F_{2}$ show similar diurnal behaviour during the magnetically-quiet first day (4 April). Both quantities increase sharply in the early morning, reach their maximum around noon, maintain relatively high values in the afternoon, and then decrease steadily in the evening hours. As seen in Fig.3C, the F2 peak height $\left(h_{m} F_{2}\right)$ is clearly in anti-phase with the peak density $\left(N_{m} F_{2}\right)$ variation during that day.

In the presented case, a geomagnetic storm started shortly after 09:00UT on 5 April 2010 and the main phase continued until about 16:00UT. During that time both TEC and $f_{o} F_{2}$ increased substantially, peaking at 12:00UT and then again at around 17:00UT. The F2 peak height, $h_{m} F_{2}$, increased steadily throughout the day, starting from a minimum of $250 \mathrm{~km}$ in the morning and reaching $400 \mathrm{~km}$ at midnight. The next day (6 April) was characterised with the development of a negative phase, i.e. depleted TEC and $f_{o} F_{2}$, up until 16:00UT when another, second step of the storm led again to pronounced TEC and $f_{o} F_{2}$ increase between 16:00UT and 22:00UT. The negative phase was well pronounced throughout the following day.

The operational reconstruction of the electron density profile (Fig.3, top panel) was unproblematic most of the time, with only a few exceptions when the digisonde failed to deliver autoscaled values or the TEC values were too low to produce realistic topside profiles (cf. the white stripes). The plot clearly shows the increased electron density and topside plasma scale height during the positive phase of the storm (5 April) and the depleted ionosphere during the recovery phase $(6$ and 7 April). Note the sharp increase of the scale height and the peak density during the second step of the storm (in the evening of 6 April), finely represented in the plot. 

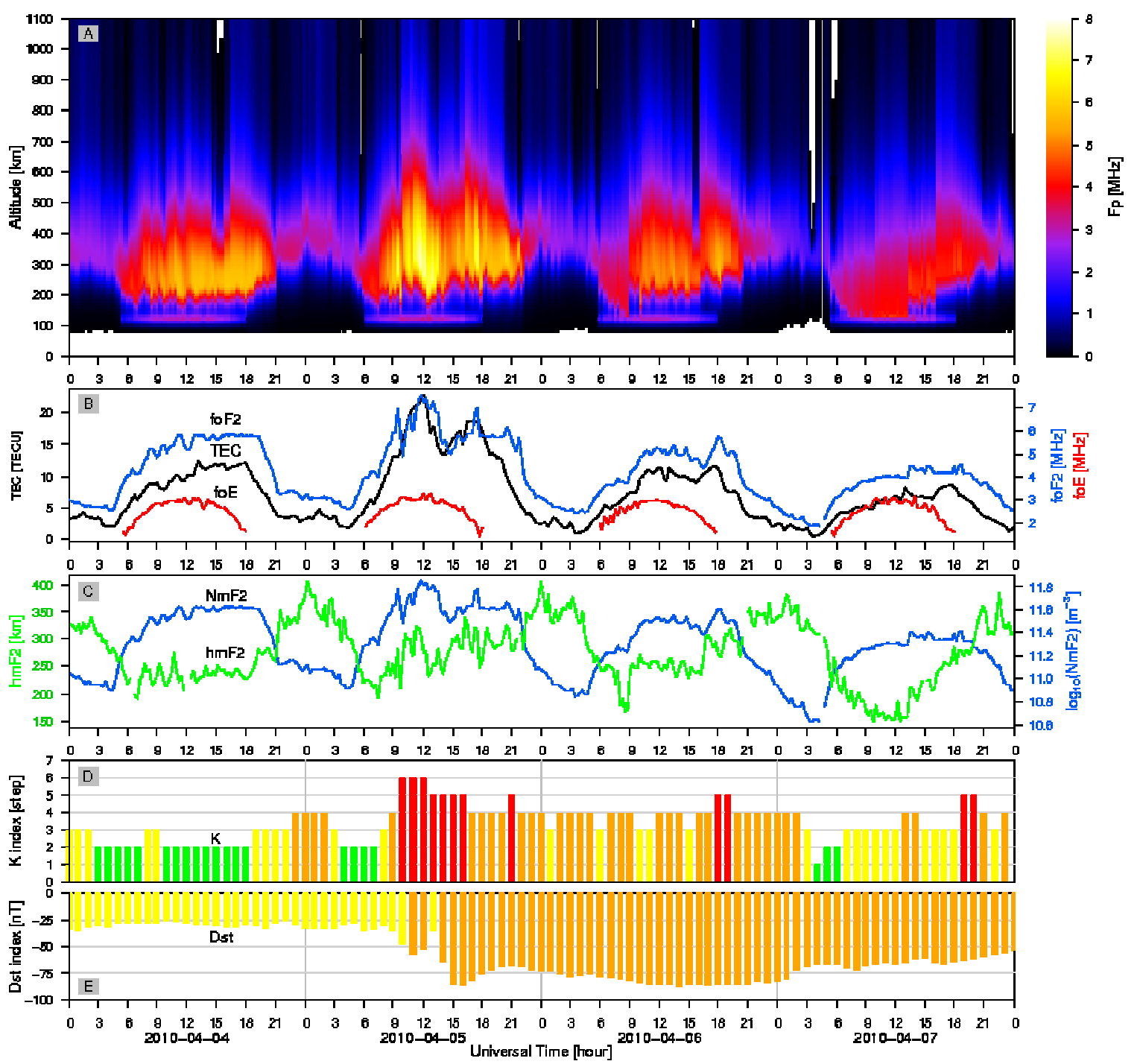

Fig.3. Real-time reconstruction of the electron density profile, Dourbes $\left(4.6^{\circ} \mathrm{E}, 50.1^{\circ} \mathrm{N}\right)$ during the storm time period 05-07 April 2010. Top panel (A): profilograms showing the reconstructed electron density (or plasma frequency) profiles as function of height and time. Panel (B): GPS TEC and ionosonde measurements of the critical frequencies $f_{o} F_{2}$ and $f_{o} E$. Panel (C): Ionosonde measurements of the peak density $\left(N_{m} F_{2}\right)$ and height $\left(h_{m} F_{2}\right)$. Panel (D): Local index $\mathrm{K}$ estimation in real time from Dourbes magnetic observatory measurements. Panel (E): Dst index in real time, estimation based on the Lund Dst model (Lundstedt et al., 2002).

The reliability of the digisonde's autoscaling process is obviously very important for the smooth operation of LIEDR. A recent overall assessment of the autoscaling algorithms performance, including ARTIST/NHPC, has found (Bamford et al., 2008) that the auto-scaled characteristics are within acceptable pre-defined error bounds for more than $80 \%$ of the time $\left(90 \%\right.$ for $f_{o} F_{2}$ and $M_{3000} F_{2}$ ) and that the geomagnetic conditions have a relatively small effect on the auto-scaling performance (a major cause of error being the truncation of automatic layer traces due to broadcast interference).

LIEDR allows for various operational extensions such as ionospheric slab thickness monitoring and forecast of ionospheric parameters. 

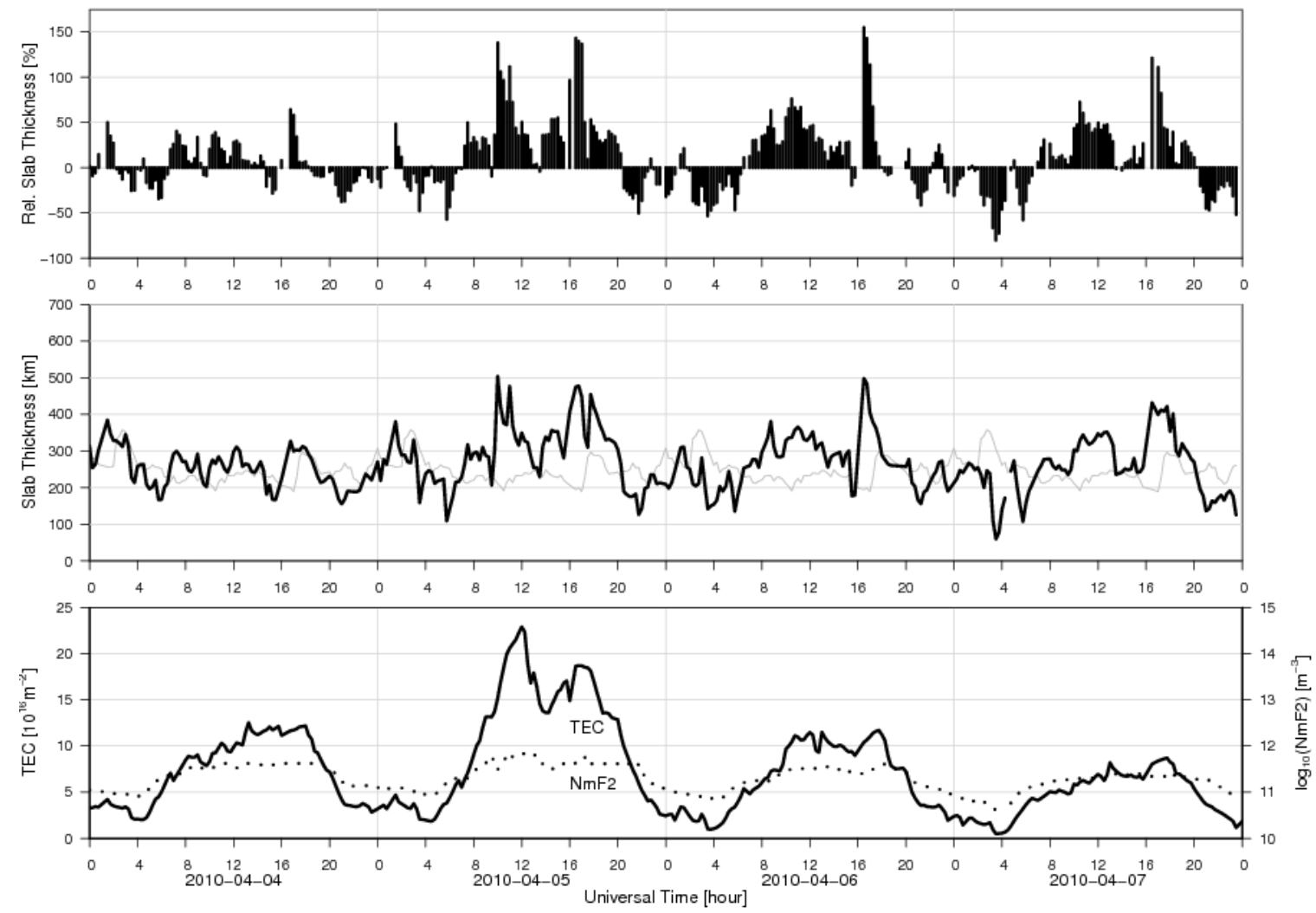

Fig.4. Real-time estimation of the ionospheric slab thickness at Dourbes $\left(4.6^{\circ} \mathrm{E}, 50.1^{\circ} \mathrm{N}\right)$. Top panel: Relative deviation (in percentage) of the slab thickness from its 27-day medians. Middle panel: Absolute slab thickness (solid black curve) and 27-day running median (grey curve). Bottom panel: GPS TEC and F2 peak density $\left(N_{m} F_{2}\right)$ measurements.

The ionospheric slab thickness, $\tau=T E C / N_{m} F_{2}$, offers substantial information on the shape of the electron density profile, the neutral and ionospheric temperatures/gradients, and the ionospheric composition and dynamics in general (Stankov and Warnant, 2009). To aid the research, a real-time monitor of the local slab thickness variations has been developed. As an example, we present here results obtained during the same storm period, 5-7 April 2010 (Fig.4). The figure shows the slab thickness values and their percentage deviations from the latest 27-day running medians, together with the raw TEC and $N_{m} F_{2}$ measurements used for the calculations. The focus is on the slab thickness percentage deviations (Fig.4, top panel) as they provide a convenient summary of the current ionospheric situation by comparison with its regular state. In the here presented results, a strong increase of about $150 \%$, relative to the median values, is observed during the storm's main phase, followed by a substantial decrease (about 50\%) during night. This behaviour is repeated in the following 48 hours, although on a smaller scale. It should be noted that, in this case, the slab thickness variation is due to the stronger response of TEC rather than the $N_{m} F_{2}$ (Fig.4, bottom panel). Raw data were used "as is" to demonstrate the exact conditions encountered during the LIEDR system operation.

Having a comprehensive database, combined with reliable measurements of the key ionospheric parameters available in real time, provides a good opportunity for operational ionospheric forecast. A practical procedure for $f_{o} F_{2}$ and TEC forecast has been developed (Stankov et al., 2009) to use the infrastructure set up for LIEDR. In this forecast procedure, the temporal behaviour of the ionospheric characteristics is considered as composed of a periodic component and a random component. The periodic component represents the average (undisturbed) behaviour, whereas the random component reflects on the variations caused by changes in the solar/geomagnetic activity. The median behaviour 
is predicted by using the latest values obtained from real-time measurements and by estimating the forward gradient based on the median behaviour over the past 27 days. The median prediction is then corrected for the influence of the 'background' level of geomagnetic activity. If the forecast window overlaps a geomagnetic storm period, suitable corrections derived from empirical data (Kutiev and Muhtarov, 2003; Stankov et al., 2010) are applied.

\section{Summary and outlook}

An operational system for deducing and imaging the vertical distribution of the electron density in the local ionosphere was presented together with some preliminary results demonstrating the system's operational capabilities. LIEDR offers an easy access to current and historical images and data records concerning the local ionospheric behaviour. The growing availability of GNSS TEC and ionosonde measurements, combined with the demonstrated ability to run the system in real time, gives both the approach and the system much potential.

Possible applications include: testing and developing various ionosphere-plasmasphere models, facilitating ionospheric data assimilation and tomography applications, optimising radio system and GNSS positioning operations, investigating ionospheric storms and other space-weather studies. For example, the ionospheric propagation delays can be estimated and corrected much easier if the electron density profile is available at a nearby location on a real-time basis. With the help of the other companion developments (geomagnetic activity and slab thickness monitoring), LIEDR offers opportunities for gaining a deeper understanding of the physical processes (and the drivers behind these processes) in the local ionosphere. Also, both the input data and the reconstruction results can be used for validation purposes in ionospheric models, maps, and services. Distribution and bounds of physically-possible model-driving parameters and solutions can be investigated. With the new wealth of prompt data, it is nowadays imperative that effective (data assimilation and tomography) methods be developed for use in ionospheric modelling and research.

Any operational nowcasting or forecasting system relies on developing and improving the techniques and methods. In this sense, the profile reconstruction technique is further being developed to incorporate variable scale height profilers. Also, the implementation of a short-term ionospheric forecast is under way.

\section{Acknowledgements}

The authors thank I. Kutiev for the useful discussions and G. Crabbe for the technical support. This work is funded by the Royal Meteorological Institute (RMI) via the Belgian Solar-Terrestrial Centre of Excellence (STCE). The solar activity index data is provided by the Belgian Solar Influences Data Centre (SIDC) and the US National Oceanic and Atmospheric Administration (NOAA). The Lund Dst model, developed at the Swedish Institute of Space Physics (IRF), is used for the Dst index calculations. Real time solar wind data from the Advanced Composition Explorer (ACE) satellite are provided by the NOAA Space Weather Prediction Center.

\section{References}

Bamford, R.A., Stamper, R., Cander, L.R. A comparison between the hourly autoscaled and manually scaled characteristics from the Chilton ionosonde from 1996 to 2004. Radio Sci. 43, RS1001, doi:10.1029/2005RS003401, 2008.

Buresova, D., Nava, B., Galkin, I., Angling, M., Stankov, S. M., Coisson, P. Data ingestion and assimilation in ionospheric models. Ann. Geophys. 52(3/4), 235-253, 2009.

Di Giovanni, G., Radicella, S.M. An analytical model of the electron density profile in the ionosphere, Adv. Space Res., 10(11), 27-30, 1990.

Hargreaves, J.K. The Solar-Terrestrial Environment. Cambridge University Press, Cambridge, 1992.

Jodogne, J.C., Stankov, S.M. Ionosphere-plasmasphere response to geomagnetic storms studied with the RMI-Dourbes comprehensive database. Ann. Geophys. 45(5), 629-647, 2002.

Klobuchar, J. Real-time ionospheric science: the new reality. Radio Sci. 32(5), 1943-1952, 1997. 
Kutiev, I., Stankov, S.M., Marinov, P., Analytical expression of $\mathrm{O}^{+}-\mathrm{H}^{+}$transition surface for use in IRI. Adv. Space Res. 14(12), 135-138, 1994.

Kutiev, I., Muhtarov, P. Empirical modeling of global ionospheric foF2 response to geomagnetic activity. J. Geophys. Res. 108(A1), 1021, 2003.

Kutiev, I., Muhtarov, P., Andonov, B., Warnant, R. Hybrid model for nowcasting and forecasting the K index. J. Atmos. Solar-Terr. Phys. 71(5), 589-596, 2009.

Lundstedt, H., Gleisner, H., Wintoft, P. Operational forecasts of the geomagnetic Dst index, Geophys. Res. Lett., 29(24), 2181, 2002.

Rawer, K. Replacement of the present sub-peak plasma density profile by a unique expression. Adv. Space Res. 2, 183-190, 1983.

Rawer, K. Synthesis of ionospheric electron density profiles with Epstein functions. Adv. Space Res. 8(4), 191-199, 1988.

Rees, D., Fuller-Rowell, T.J., The response of the thermosphere and ionosphere to magnetosphere forcing. Phil. Trans. R. Soc. London, A328, 139-171, 1989.

Reinisch, B.W. Modern ionosondes. in: Kohl, H., Rüster, R., Schlegel, K. (eds.), Modern Ionospheric Science, European Geophysical Society, Katlenburg-Lindau, 440-458, 1996.

Reinisch, B.W., Huang, X., Galkin, I.A., Paznukhov, V., Kozlov, A. Recent advances in real-time analysis of ionograms and ionospheric drift measurements with digisondes. J. Atmos. Solar-Terr. Phys. 67(12), 1054-1062, 2005.

Reinisch, B.W., Nsumei, P., Huang, X., Bilitza, D.K. Modeling the F2 topside and plasmasphere for IRI using IMAGE/RPI and ISIS data. Adv. Space Res. 39(5), 731-738, 2007.

Sauli, P., Mosna, Z., Boska, J., Kouba, D., Lastovicka, J., Altadill, D. Comparison of true-height electron density profiles derived by POLAN and NHPC methods. Stud. Geophys. Geod. 51(3), 449-459, 2007.

Schunk, R.W., Scherliess, L., Sojka, J.J., et al. Global assimilation of ionospheric measurements (GAIM). Radio Sci. 39, RS1S02, doi:10.1029/2002RS002794, 2004.

Stamper, R., Belehaki, A., Buresova, D., Cander, L., Kutiev, I., Pietrella, M., Stanislawska, I., Stankov, S., Tsagouri, I., Tulunay, Y., Zolesi, B. Nowcasting, forecasting and warning for ionospheric propagation. Ann. Geophys. 47(2/3), 957-984, 2004.

Stankov, S.M. Empirical modelling of ion transition levels based on satellite in-situ measurements. C. R. Acad. Bul. Sci. 55(1), 35-40, 2002a.

Stankov, S. M. Evaluation of analytical ionospheric models used in electron density profile reconstruction. Acta Geod. Geophys. Hung. 37, 385- 401, 2002b.

Stankov, S.M., Jakowski, N., Heise, S., Muhtarov, P., Kutiev, I., Warnant, R. A new method for reconstruction of the vertical electron density distribution in the upper ionosphere and plasmasphere. J. Geophys. Res. 108(A5), 1164, doi:10.1029/2002JA009570, 2003.

Stankov, S.M., Jakowski, N., Heise, S. Reconstruction of ion and electron density profiles from spacebased measurements of the upper electron content. Planet. Space Sci. 53(9), 945-957, doi:10.1016/j.pss.2005.04.008, 2005.

Stankov, S.M., Jakowski, N. Topside ionospheric scale height analysis and modelling based on radio occultation measurements. J. Atmos. Solar-Terr. Phys. 68 (2), 134-162, doi:10.1016/j.jastp.2005.10.003, 2006.

Stankov, S.M., Warnant, R. Ionospheric slab thickness - analysis, modelling and monitoring. Adv. Space Res. 44(11), 1295-1303, doi:10.1016/j.asr.2009.07.010, 2009.

Stankov, S.M., Warnant, R., Kozarev, R. On the TEC short-term forecast with corrections based on the average ionospheric response to background and storm-time geomagnetic conditions. Geophys. Res. Abstr. 11, Abs. No. EGU2009-13283, 2009.

Stankov, S.M., Stegen, K., Warnant, R. Seasonal variations of storm-time TEC at European middle latitudes, Adv. Space Res. 46(10), 1318-1325, doi:10.1016/j.asr.2010.07.017, 2010.

Titheridge, J. The real height analysis of ionograms: A generalized formulation. Radio Sci. 23(5), 831-849, 1988.

Warnant, R., Pottiaux, E. The increase of the ionospheric activity as measured by GPS. Earth Planets Space 52(11), 1055-1060, 2000. 